\title{
Dupilumab with Topical Corticosteroids Provides Rapid and Sustained Improvement in Adults with Moderate-to-Severe Atopic Dermatitis Across Anatomic Regions Over 52 Weeks
}

Andrew Blauvelt · Marjolein de Bruin-Weller • Eric L. Simpson •

Zhen Chen · Annie Zhang · Brad Shumel (D)

Received: October 5, 2021 / Published online: November 22, 2021

(C) The Author(s) 2021

\section{ABSTRACT}

Introduction: In a 52-week, phase 3 clinical trial (LIBERTY AD CHRONOS) in adult patients with moderate-to-severe atopic dermatitis (AD), dupilumab in combination with topical corticosteroids (TCS) resulted in a significant improvement in overall Eczema Area and Severity Index (EASI) compared with placebo plus TCS. In a post hoc analysis, dupilumab significantly improved the overall extent and severity of AD across four anatomic regions (head and neck, trunk, upper extremities, lower extremities) over 16 weeks. However, as AD severity and presentation may vary by body region, this analysis sought to determine

A. Blauvelt

Oregon Medical Research Center, Portland, OR, USA

M. de Bruin-Weller

National Expertise Center of Atopic Dermatitis, Department of Dermatology and Allergology,

University Medical Center Utrecht, Utrecht, The

Netherlands

E. L. Simpson

Department of Dermatology, Oregon Health and

Science University, Portland, OR, USA

Z. Chen · B. Shumel $(\bowtie)$

Regeneron Pharmaceuticals, Inc., 777 Old Saw Mill

River Road, Tarrytown, NY 10591, USA

e-mail: brad.shumel@regeneron.com

A. Zhang

Sanofi Genzyme, Cambridge, MA, USA whether there are regional variations in dupilumab efficacy.

Methods: Using data from the LIBERTY AD CHRONOS study, we performed a post hoc analysis of the mean percentage change in individual EASI signs (erythema, infiltration/papulation, excoriation, lichenification) from baseline through week 52 across four anatomic regions (head and neck, trunk, upper extremities, lower extremities).

Results: Dupilumab plus TCS, compared with placebo plus TCS, significantly improved the severity of all individual $\mathrm{AD}$ signs to a similar extent across the four anatomic regions. Significant improvements in each sign were seen early, within the first 2-4 weeks of treatment, and were sustained through week 52 across all regions.

Conclusions: In adult patients with moderateto-severe $\mathrm{AD}$, treatment with dupilumab resulted in rapid and sustained improvement in the signs of $\mathrm{AD}$ across all anatomic regions.

Trial registration: LIBERTY AD CHRONOS (NCT02260986).

Keywords: Anatomic regions; Atopic dermatitis; Atopic eczema; Contact dermatitis; Cytokines; Dermatology; Dupilumab; EASI; Facial rash; Immunology; Signs 


\section{Key Summary Points}

Why carry out this study?

In phase 3 trials in adults with moderateto-severe atopic dermatitis (AD), treatment with dupilumab resulted in a substantial reduction in overall disease severity.

Dupilumab was equally efficacious in reducing $\mathrm{AD}$ severity in four anatomic regions measured by Eczema Area and Severity Index (EASI) over 16 weeks.

As $\mathrm{AD}$ severity and presentation may vary by body region, the objective of this analysis was to characterize the efficacy of dupilumab with respect to individual $A D$ signs across four anatomic regions as assessed by EASI over 52 weeks in adults with moderate-to-severe AD.

What was learned from the study?

Dupilumab demonstrated rapid and sustained improvement, maintained through 52 weeks, in the individual signs of AD across all anatomic regions in adults with moderate-to-severe AD.

\section{INTRODUCTION}

The presentation of atopic dermatitis (AD) can differ by anatomic region, with morphologic variants showing predispositions for certain body areas [1]. Facial regions and areas with skin flexures may show a range of $\mathrm{AD}$ signs [1]. Sensitive areas with thinner skin, such as the head and neck, may be more susceptible to irritants and allergens, and vulnerable to skinthinning effects of topical corticosteroids (TCS) [2].

Dupilumab, a monoclonal antibody that blocks the shared receptor component for interleukin (IL)-4 and IL-13 [3-6], has demonstrated significant efficacy and an acceptable safety profile in patients with moderate-to-severe $\mathrm{AD}$ and other type 2 inflammatory diseases [7-16].

In the 52-week LIBERTY AD CHRONOS study, dupilumab plus TCS, compared with placebo plus TCS, resulted in a significant improvement in overall Eczema Area and Severity Index (EASI) [10]. In a 16-week analysis of four clinical trials in adults with moderate-tosevere $\mathrm{AD}$, including LIBERTY AD CHRONOS, dupilumab plus TCS compared with placebo plus TCS significantly improved the extent and severity of $\mathrm{AD}$ across four anatomic regions (head and neck, trunk, upper extremities, lower extremities) as assessed by EASI [17].

Although no safety signal was detected during randomized clinical trials of dupilumab in $\mathrm{AD}$, a number of individual case reports and limited case series describe a new-onset or acute worsening of facial rash associated with dupilumab treatment [18-28]. Reported clinical signs and symptoms include erythema, edema, flushing, papulopustules, pruritus, scaling, and a burning sensation in the centrofacial area $[26,28]$. Various possible etiologies have been suggested, including corticosteroid withdrawal, rosacea, contact dermatitis, Malassezia colonization involving the face and neck, seborrheic dermatitis, photosensitivity, and alcohol-induced facial flushing [19, 20, 22-25, 28, 29].

EASI is a composite score encompassing severity of AD signs and extent of disease over four anatomic regions: head/neck, upper extremities, trunk, and lower extremities. To further evaluate whether certain anatomic areas may respond in a differential manner to dupilumab therapy, we analyzed the change, over 52 weeks, in individual EASI signs (erythema, infiltration/papulation, excoriation, lichenification) across the four anatomic regions in patients included in the LIBERTY $\mathrm{AD}$ CHRONOS study.

\section{METHODS}

We performed a post hoc analysis of data from the randomized, double-blinded, placebo-controlled, multicenter, parallel group, phase 3 LIBERTY AD CHRONOS trial (NCT02260986) of 
dupilumab [10]. The full study design and patient population of LIBERTY AD CHRONOS have been previously reported [10]. Briefly, LIBERTY AD CHRONOS enrolled adult patients (at least 18 years of age) with moderate-to-severe $\mathrm{AD}$ inadequately controlled with TCS. Patients were randomized 3:1:3 to receive dupilumab $300 \mathrm{mg}$ subcutaneously (SC) once weekly (qw; with a loading dose of $600 \mathrm{mg}$ ), dupilumab $300 \mathrm{mg}$ SC every 2 weeks (q2w; with a loading dose of $600 \mathrm{mg}$ ), or placebo qw, respectively, for a period of 52 weeks. All patients received a concomitant standardized regimen of medium-potency TCS, which could be tapered, stopped, or restarted on the basis of disease activity.

LIBERTY AD CHRONOS was conducted in accordance with the provisions of the Declaration of Helsinki, the International Conference on Harmonisation Good Clinical Practice guideline (version R1), and applicable regulatory requirements. All patients provided signed written informed consent. The protocol and all relevant study forms were approved by all relevant institutional review boards and an independent ethics committee.

\section{Endpoints}

EASI is a validated measure to assess the severity and extent of $\mathrm{AD}$ [30]. The score is a composite of the investigator's assessment of the severity of four individual signs (erythema, infiltration/papulation, excoriation, and lichenification) rated on a scale of 0 (absent) through 3 (severe), and the extent of $\mathrm{AD}$ involvement assessed as a percentage of body surface area in each of four anatomic regions (head and neck, trunk, upper extremities, lower extremities) converted to a score of 0 to 6 as follows: $0(0 \%)$, 1 (1-9\%), 2 (10-29\%), 3 (30-49\%), 4 (50-69\%), 5 (70-89\%), or 6 (90-100\%).

For each region, the severity of the four signs is summated $(0-12)$ and then multiplied by the extent of involvement (0-6). Least squares (LS) mean percentage change in EASI from baseline through week 52 for each of the four AD signs was evaluated in four anatomic regions.

\section{Analysis}

Only data for patients who received the approved dupilumab dose regimen of $300 \mathrm{mg}$ q2w plus TCS, or placebo plus TCS in the study, were included in this post hoc analysis.

Endpoints were analyzed using an analysis of covariance model with baseline measurement as covariate and the treatment, randomization strata (baseline disease severity [Investigator's Global Assessment (IGA) $=3$ vs IGA = 4]), and geographical region as fixed factors.

Values after rescue medication use were set to missing. For LS mean percentage change in EASI signs, missing values were imputed using the last observation carried forward method. $p<0.05$ (two-sided tests) was regarded as significant. Since this analysis was post hoc, and not adjusted for multiplicity, the $p$ values provided in the manuscript are nominal. Statistical Analysis Software, version 9.4 (SAS Institute, Inc; Cary, NC, USA) was used for all analyses.

\section{RESULTS}

In the LIBERTY AD CHRONOS study, 740 patients were enrolled (319 dupilumab $300 \mathrm{mg}$ qw plus TCS, 106 dupilumab $300 \mathrm{mg}$ q2w plus TCS, and 315 placebo plus TCS) [10]. In the present analysis, 421 patients were included: 106 patients who received the approved adult dose regimen of dupilumab $300 \mathrm{mg} \mathrm{q} 2 \mathrm{w}$ plus TCS (median age 40.5 years; $58 \%$ male) and the 315 patients who received placebo plus TCS (median age 34.0 years; 61\% male) [10]. Baseline demographics and clinical characteristics of all patients included in LIBERTY AD CHRONOS (including overall EASI values) have been previously reported and were similar between the dupilumab plus TCS and placebo plus TCS groups [10].

At baseline, EASI for individual signs across anatomic regions was similar between the dupilumab $300 \mathrm{mg}$ q2w plus TCS and placebo plus TCS groups (Fig. 1). Dupilumab $300 \mathrm{mg}$ q2w plus TCS significantly improved each individual EASI sign in all four anatomic regions, including lichenification, the $\mathrm{AD}$ sign most resistant to treatment (Fig. 1) [31]. 

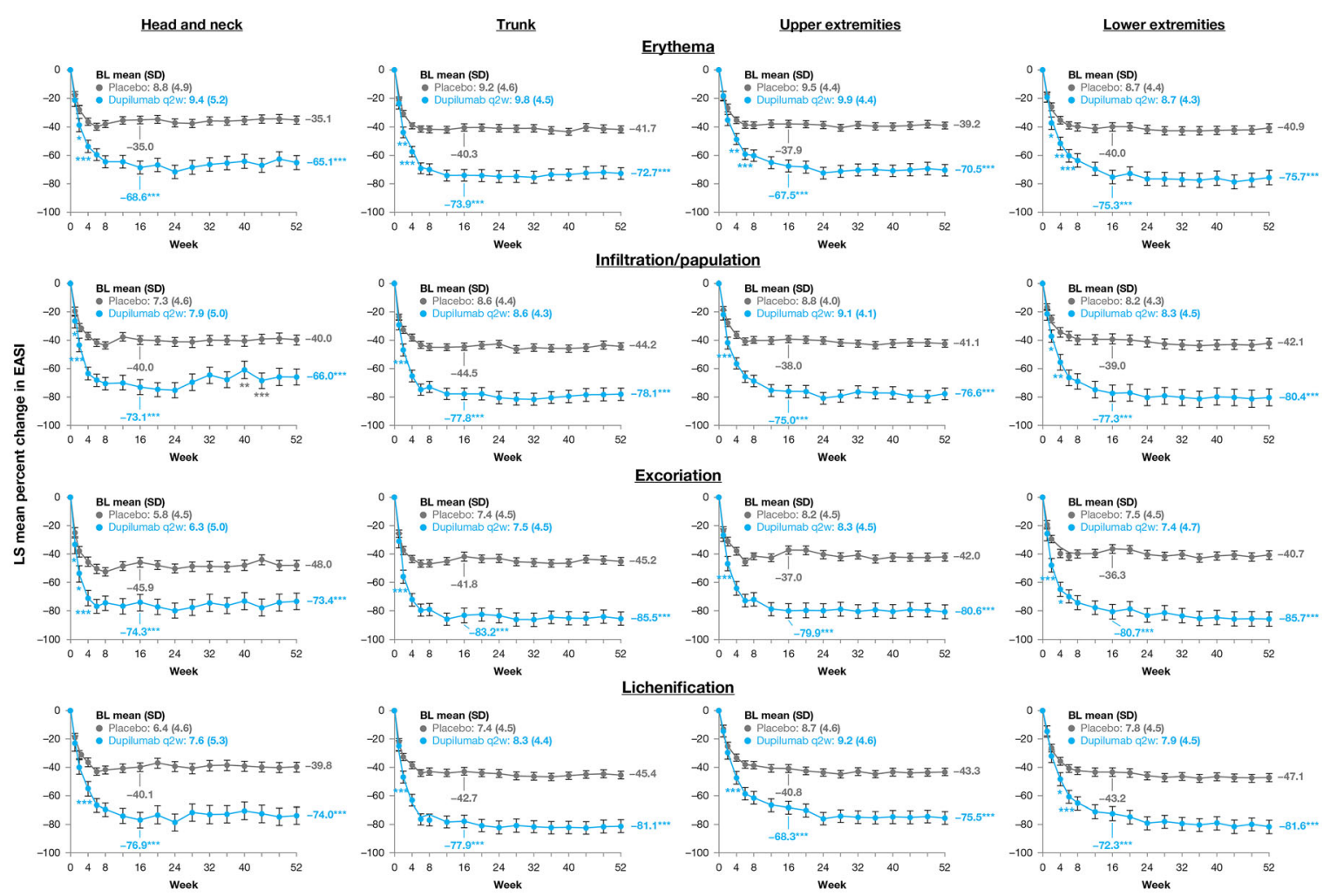

Fig. 1 LS mean percentage change in EASI for erythema, infiltration/papulation, excoriation, and lichenification from baseline to week 52 by visit in four anatomic regions.

Significant improvements were seen in most cases as early as week 2 , and in all cases as early as week 4 , with further improvements through week 16, which were maintained through week 52.

With respect to erythema, significant improvements with dupilumab plus TCS were seen in the head/neck, trunk, and lower extremities as early as week 2 and in the upper extremities by week 4 . At week 52, placebo-corrected improvements in erythema were similar in all regions, ranging from -30.0 in the head and neck region to -34.8 in the lower extremity region, although the LS mean percentage change from baseline in the dupilumab plus TCS treatment group in the head and neck region $(-65.1 \%)$ was slightly lower than the improvement observed in other regions, which ranged from $-70.5 \%$ to $-75.7 \%$. A similar pattern was also observed for the signs of
${ }^{*} p<0.05$ vs placebo; ${ }^{* *} p \leq 0.01$ vs placebo; ${ }^{* * *} p \leq 0.001$ vs placebo. $B L$ baseline, EASI Eczema and Severity Index, $L S$ least squares, $q 2 w$ every 2 weeks, $S D$ standard deviation

infiltration/papulation and excoriation in the head and neck region vis-a-vis the other three regions (i.e., numerically lower placebo-corrected differences and lower absolute mean change from baseline). A body map illustrating LS mean percentage change in EASI at week 52 for each sign in each anatomic region is displayed in Fig. 2.

\section{DISCUSSION}

Dupilumab plus TCS significantly improved the severity of individual AD signs across four anatomic regions as assessed by EASI in the randomized, placebo-controlled phase 3 LIBERTY AD CHRONOS trial in adults with moderate-tosevere $\mathrm{AD}$.

The results, which show comparable improvements across anatomic regions, confirm and extend the short-term results seen in adults 


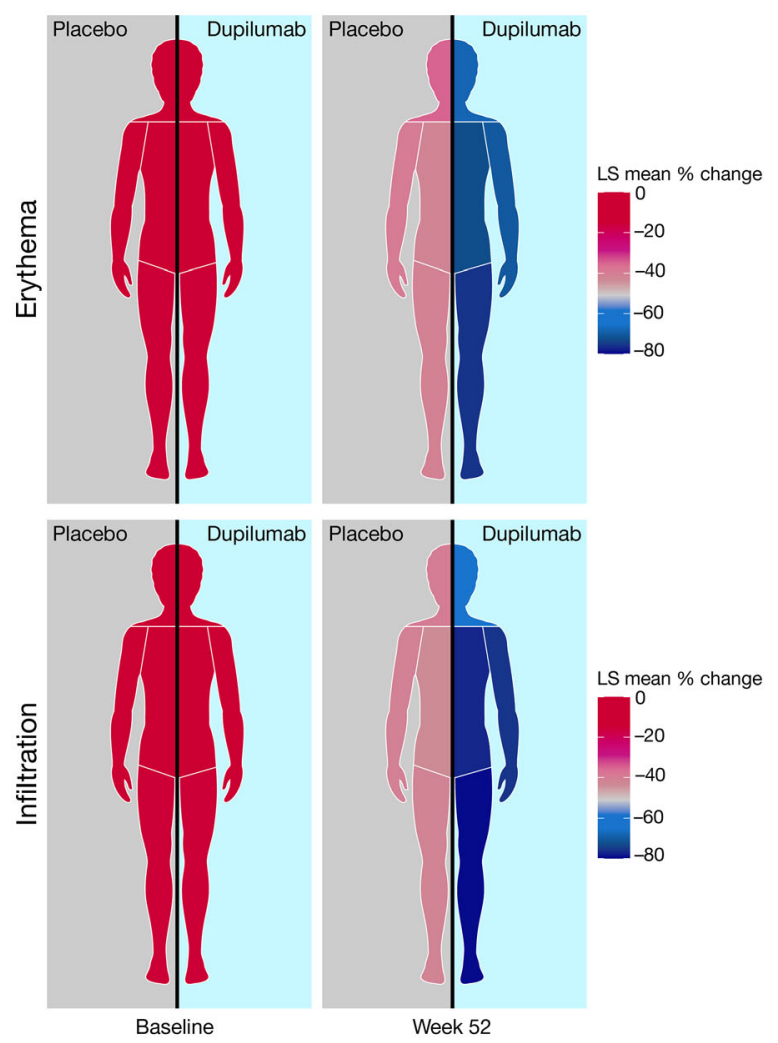

Fig. 2 Body map ${ }^{a}$ showing LS mean percentage change in EASI for erythema, infiltration/papulation, excoriation, lichenification at baseline and at week 52 in four anatomic

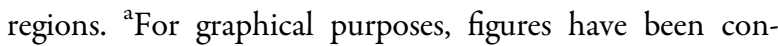
structed to represent the right side of the body being

with moderate-to-severe $\mathrm{AD}$ who received dupilumab with or without TCS [17]. Improvements in all regions and signs were seen early, after the first or second dupilumab dose, and were maintained through week 52. Dupilumab was generally well tolerated with an acceptable safety profile in the LIBERTY AD CHRONOS study [10].

Facial AD lesions may have a higher impact on a patient's quality of life than $\mathrm{AD}$ lesions in other anatomic regions [32], as it is a highly visible and an aesthetically important site. Recent studies have described new-onset or acute worsening of facial rash and posited various etiologies-many of which are presumably unrelated to dupilumab treatment-including rosacea, allergic contact dermatitis, Malassezia colonization involving the face and neck,

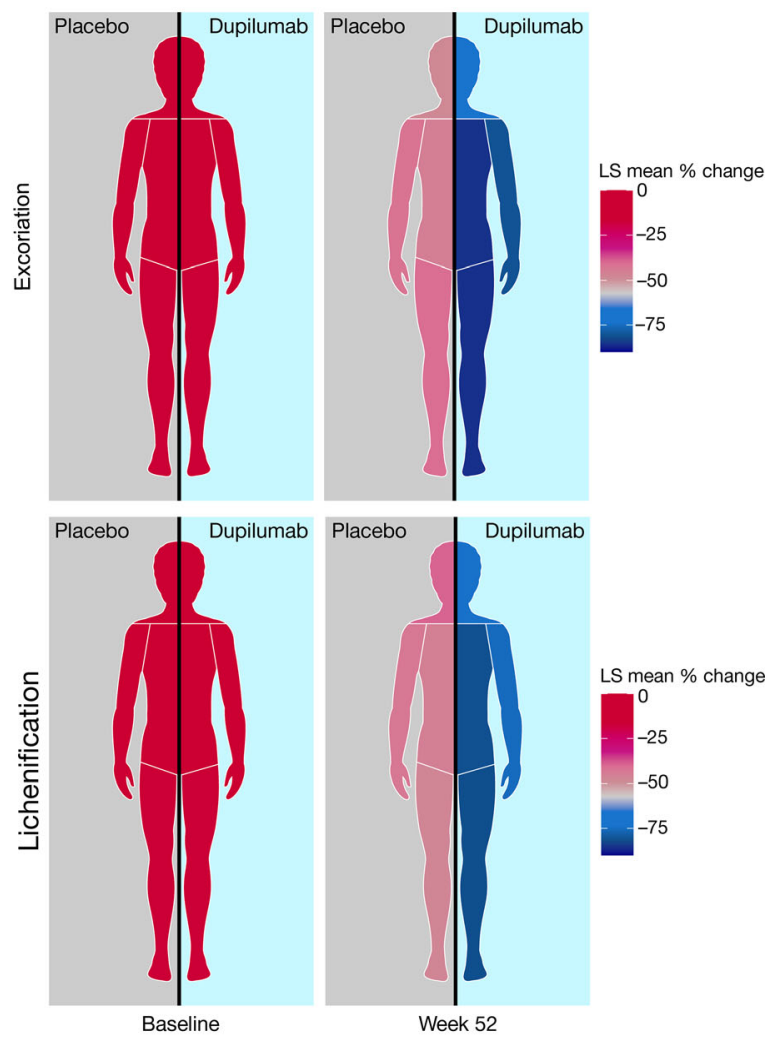

treated with placebo and the left side being treated with dupilumab. In patients receiving dupilumab, similar responses were achieved on both sides of the body. EASI Eczema Area and Severity Index, $L S$ least squares

photosensitivity, and steroid withdrawal [18-28]. The face is a common site for exacerbation by irritants and/or allergic contact dermatitis [33]. For most patients with $\mathrm{AD}$ and allergic contact dermatitis, identification and avoidance of the contact allergen is the only definitive management approach [34]. Additionally, steroid withdrawal can trigger rebound erythema in the head and neck region while fluorinated TCS can trigger rosacea or periorificial facial dermatitis [35-38]. In this analysis, improvement in erythema score for the head and neck region was comparable to, albeit slightly lower than, that seen in other regions, suggesting that instances of facial rash associated with dupilumab treatment reported in the literature are unlikely to represent $\mathrm{AD}$ lesions recalcitrant to dupilumab treatment and arise 
independently of the IL-4/IL-13 pathway. This observation may also suggest that the head and neck region is a more difficult area to treat.

A limitation is the inability to further subdivide EASI assessment parameters within each anatomic region. For example, $\mathrm{AD}$ signs in the head and neck region cannot specifically discern changes in signs in the facial region, nor can evaluation of signs in the upper extremities specifically assess changes in the hands.

\section{CONCLUSIONS}

In LIBERTY $\mathrm{AD}$ CHRONOS, a randomized, double-blinded, placebo-controlled phase 3 trial in adults with moderate-to-severe $\mathrm{AD}$, treatment with dupilumab resulted in comparable improvements in $\mathrm{AD}$ signs (erythema, infiltration/papulation, excoriation, and lichenification) across four anatomic regions (head and neck, trunk, upper extremities, lower extremities). Improvements in all signs in each anatomic region were seen early, after the first or second dupilumab dose, and were sustained through week 52. Dupilumab was generally well tolerated with an acceptable safety profile.

\section{ACKNOWLEDGEMENTS}

The authors thank the patients and their families for their participation in these studies; their colleagues for their support; and Linda Williams (Regeneron Pharmaceuticals, Inc.), El-Bdaoui Haddad, Tracy Chew, and Adriana Mello (Sanofi Genzyme) for their contributions.

Funding. This research was sponsored by Sanofi and Regeneron Pharmaceuticals, Inc. The journal Rapid Service Fee was sponsored by Sanofi Genzyme and Regeneron Pharmaceuticals, Inc.

Medical Writing and Editorial Assistance. Medical writing and editorial assistance were provided by Ekaterina Semenova, PhD, of Excerpta Medica, and was funded by Sanofi Genzyme and Regeneron Pharmaceuticals, Inc. according to the Good Publication Practice guideline.

Authorship. All named authors meet the International Committee of Medical Journal Editors (ICMJE) criteria for authorship for this article, take responsibility for the integrity of the work as a whole, and have given their approval for this version to be published.

Author Contributions. AB, BS contributed to study concept and design. AB, MdeB-W, ELS acquired data. ZC conducted the statistical analyses of the data. All authors interpreted the data, provided critical feedback on the manuscript, approved the final manuscript for submission, and are accountable for the accuracy and integrity of the manuscript.

Disclosures. Andrew Blauvelt is a scientific adviser, clinical study investigator for AbbVie, Abcentra, Aligos Therapeutics, Almirall, Amgen, Arcutis Biotherapeutics, Arena Pharmaceuticals, Aslan Pharmaceuticals, Athenex, Boehringer Ingelheim, Bristol Myers Squibb, Dermavant, Eli Lilly, Evommune, Forté, Galderma, Incyte, Janssen, Landos Biopharma, LEO Pharma, Novartis, Pfizer, RAPT Therapeutics, Regeneron Pharmaceuticals, Inc., Sanofi Genzyme, Sun Pharma, UCB.

Marjolein de Bruin-Weller is a consultant, advisory board member, and/or speaker for AbbVie, Almirall, Arena Pharmaceuticals, Aslan Pharmaceuticals, Eli Lilly, Galderma, Janssen, LEO Pharma, Pfizer, Regeneron Pharmaceuticals, Inc., Sanofi Genzyme, UCB.

Eric L. Simpson has received personal fees from AbbVie, Boehringer Ingelheim, Collective Acumen, Eli Lilly, Forté Biosciences, Incyte, Janssen, Kyowa Hakko Kirin, LEO Pharma, Novartis, Ortho Dermatologics, Pfizer, Pierre Fabre Dermo-Cosmetics, Regeneron Pharmaceuticals, Inc., Roivant Sciences, Sanofi, Valeant; received grants and is a Principal Investigator for AbbVie, Eli Lilly, Incyte, Kyowa Hakko Kirin, LEO Pharma, Merck, Novartis, Pfizer, Regeneron Pharmaceuticals, Inc., Sanofi, Tioga Pharmaceuticals, Vanda Pharmaceuticals.

Zhen Chen and Brad Shumel are employees and shareholders of Regeneron Pharmaceuti- 
cals, Inc.

Annie Zhang is an employee of Sanofi Genzyme and may hold stock and/or stock options in the company.

Compliance with Ethics Guidelines. The trial was conducted in accordance with the ethical principles outlined in the Declaration of Helsinki and with the International Council for Harmonisation guidelines for good clinical practice and applicable regulatory requirements. The studies were approved by the appropriate institutional ethics committees at each participating institution. All patients provided written consent/assent, and at least one parent or guardian for each adolescent patient provided written informed consent.

Data Availability. For LIBERTY AD CHRONOS (NCT02260986): Qualified researchers may request access to study documents (including the clinical study report, study protocol with any amendments, blank case report form, statistical analysis plan) that support the methods and findings reported in this manuscript. Individual anonymized participant data will be considered for sharing once the product and indication have been approved by major health authorities (e.g., FDA, EMA, PMDA, etc.), if there is legal authority to share the data and there is not a reasonable likelihood of participant re-identification. Submit requests to https://vivli.org/.

Open Access. This article is licensed under a Creative Commons Attribution-NonCommercial 4.0 International License, which permits any non-commercial use, sharing, adaptation, distribution and reproduction in any medium or format, as long as you give appropriate credit to the original author(s) and the source, provide a link to the Creative Commons licence, and indicate if changes were made. The images or other third party material in this article are included in the article's Creative Commons licence, unless indicated otherwise in a credit line to the material. If material is not included in the article's Creative Commons licence and your intended use is not permitted by statutory regulation or exceeds the permitted use, you will need to obtain permission directly from the copyright holder. To view a copy of this licence, visit http://creativecommons.org/licenses/by$\mathrm{nc} / 4.0 /$.

\section{REFERENCES}

1. Girolomoni G, de Bruin-Weller M, Aoki V, et al. Nomenclature and clinical phenotypes of atopic dermatitis. Ther Adv Chronic Dis. 2021;12: 20406223211002980.

2. Draelos ZD. Use of topical corticosteroids and topical calcineurin inhibitors for the treatment of atopic dermatitis in thin and sensitive skin areas. Curr Med Res Opin. 2008;24(4):985-94.

3. Macdonald LE, Karow M, Stevens S, et al. Precise and in situ genetic humanization of $6 \mathrm{Mb}$ of mouse immunoglobulin genes. Proc Natl Acad Sci U S A. 2014;111(14):5147-52.

4. Murphy AJ, Macdonald LE, Stevens S, et al. Mice with megabase humanization of their immunoglobulin genes generate antibodies as efficiently as normal mice. Proc Natl Acad Sci U S A. 2014;111(14):5153-8.

5. Gandhi NA, Pirozzi G, Graham NMH. Commonality of the IL-4/IL-13 pathway in atopic diseases. Expert Rev Clin Immunol. 2017;13(5):425-37.

6. Le Floc'h A, Allinne J, Nagashima K, et al. Dual blockade of IL-4 and IL-13 with dupilumab, an IL$4 \mathrm{R} \alpha$ antibody, is required to broadly inhibit type 2 inflammation. Allergy. 2020;75(5):1188-204.

7. Beck LA, Thaçi D, Hamilton JD, et al. Dupilumab treatment in adults with moderate-to-severe atopic dermatitis. N Engl J Med. 2014;371(2):130-9.

8. Thaçi D, Simpson EL, Beck LA, et al. Efficacy and safety of dupilumab in adults with moderate-tosevere atopic dermatitis inadequately controlled by topical treatments: a randomised, placebo-controlled, dose-ranging phase $2 \mathrm{~b}$ trial. Lancet. 2016;387(10013):40-52.

9. Simpson EL, Bieber T, Guttman-Yassky E, et al. Two phase 3 trials of dupilumab versus placebo in atopic dermatitis. N Engl J Med. 2016;375(24):2335-48.

10. Blauvelt A, de Bruin-Weller M, Gooderham M, et al. Long-term management of moderate-to-severe atopic dermatitis with dupilumab and concomitant topical corticosteroids (LIBERTY AD CHRONOS): a 1-year, randomised, double-blinded, placebo- 
controlled, phase 3 trial. Lancet. 2017;389(10086): 2287-303.

11. de Bruin-Weller $\mathrm{M}$, Thaçi $\mathrm{D}$, Smith $\mathrm{CH}$, et al. Dupilumab with concomitant topical corticosteroid treatment in adults with atopic dermatitis with an inadequate response or intolerance to ciclosporin A or when this treatment is medically inadvisable: a placebo-controlled, randomized phase III clinical trial (LIBERTY AD CAFE). $\mathrm{Br} \quad \mathrm{J}$ Dermatol. 2018;178(5):1083-101.

12. Worm M, Simpson EL, Thaçi D, et al. Efficacy and safety of multiple dupilumab dose regimens after initial successful treatment in patients with atopic dermatitis: a randomized clinical trial. JAMA Dermatol. 2020;156(2):131-43.

13. Paller AS, Bansal A, Simpson EL, et al. Clinically meaningful responses to dupilumab in adolescents with uncontrolled moderate-to-severe atopic dermatitis: post-hoc analyses from a randomized clinical trial. Am J Clin Dermatol. 2020;21(1):119-31.

14. Castro M, Corren J, Pavord ID, et al. Dupilumab efficacy and safety in moderate-to-severe uncontrolled asthma. N Engl J Med. 2018;378(26): 2486-96.

15. Bachert C, Mannent L, Naclerio RM, et al. Effect of subcutaneous dupilumab on nasal polyp burden in patients with chronic sinusitis and nasal polyposis: a randomized clinical trial. JAMA. 2016;315(5): 469-79.

16. Hirano I, Dellon ES, Hamilton JD, et al. Efficacy of dupilumab in a phase 2 randomized trial of adults with active eosinophilic esophagitis. Gastroenterology. 2020;158(1):111-22.e10.

17. Blauvelt A, Rosmarin D, Bieber $\mathrm{T}$, et al. Improvement of atopic dermatitis with dupilumab occurs equally well across different anatomical regions: data from phase III clinical trials. Br J Dermatol. 2019;181(1):196-7.

18. Dalia Y, Johnson SM. Case report: first reported case of facial rash after dupilumab therapy. Pract Dermatol. 2018;25-6.

19. Soria A, Du-Thanh A, Seneschal J, et al. Development or exacerbation of head and neck dermatitis in patients treated for atopic dermatitis with dupilumab. JAMA Dermatol. 2019;155(11):1312-5.

20. Zhu GA, Chen JK, Chiou A, Ko J, Honari G. Assessment of the development of new regional dermatoses in patients treated for atopic dermatitis with dupilumab. JAMA Dermatol. 2019;155(7): 850-2.
21. de Beer FSA, Bakker DS, Haeck I, et al. Dupilumab facial redness: positive effect of itraconazole. JAAD Case Rep. 2019;5(1):888-91.

22. de Wijs LEM, Nguyen NT, Kunkeler ACM, Nijsten T, Damman J, Hijnen DJ. Clinical and histopathological characterization of paradoxical head and neck erythema in patients with atopic dermatitis treated with dupilumab: a case series. $\mathrm{Br} \mathrm{J}$ Dermatol. 2020;183(4):745-9.

23. Muzumdar S, Zubkov M, Waldman R, DeWane ME, Wu R, Grant-Kels JM. Characterizing dupilumab facial redness in children and adolescents: a singleinstitution retrospective chart review. J Am Acad Dermatol. 2020;83(5):1520-1.

24. Waldman RA, DeWane ME, Sloan B, Grant-Kels JM. Characterizing dupilumab facial redness: a multiinstitution retrospective medical record review. J Am Acad Dermatol. 2020;82(1):230-2.

25. Igelman SJ, Na C, Simpson EL. Alcohol-induced facial flushing in a patient with atopic dermatitis treated with dupilumab. JAAD Case Rep. 2020;6(2): 139-40.

26. Quint T, Brunner PM, Sinz C, et al. Dupilumab for the treatment of atopic dermatitis in an Austrian cohort-real-life data shows rosacea-like folliculitis. J Clin Med. 2020;9(4):1241.

27. Bax CE, Khurana MC, Treat JR, Castelo-Soccio L, Rubin AI, McMahon PJ. New-onset head and neck dermatitis in adolescent patients after dupilumab therapy for atopic dermatitis. Pediatr Dermatol. 2021;38(2):390-4.

28. Jo CE, Finstad A, Georgakopoulos JR, Piguet V, Yeung J, Drucker AM. Facial and neck erythema associated with dupilumab treatment: a systematic review. J Am Acad Dermatol. 2021;84(5):1339-47.

29. Suresh R, Murase JE. The role of expanded series patch testing in identifying causality of residual facial dermatitis following initiation of dupilumab therapy. JAAD Case Rep. 2018;4(9):899-904.

30. Hanifin JM, Thurston M, Omoto M, Cherill R, Tofte SJ, Graeber M. The eczema area and severity index (EASI): assessment of reliability in atopic dermatitis. EASI Evaluator Group Exp Dermatol. 2001;10(1): $11-8$.

31. Barbarot S, Wollenberg A, Silverberg JI, et al. Dupilumab provides rapid and sustained improvement in SCORAD outcomes in adults with moderate-to-severe atopic dermatitis: combined results of four randomized phase 3 trials. J Dermatolog Treat. 2020;1-12. https://doi.org/10.1080/09546634. 2020.1750550 . 
32. Holm JG, Agner T, Clausen M-L, Thomsen SF. Quality of life and disease severity in patients with atopic dermatitis. J Eur Acad Dermatol Venereol. 2016;30(10):1760-7.

33. Tada J, Toi Y, Arata J. Atopic dermatitis with severe facial lesions exacerbated by contact dermatitis from topical medicaments. Contact Dermatitis. 1994;31(4):261-3.

34. Pootongkam S, Nedorost S. Allergic contact dermatitis in atopic dermatitis. Curr Treat Options Allergy. 2014;1:329-36.

35. Ference JD, Last AR. Choosing topical corticosteroids. Am Fam Physician. 2009;79(2):135-40.
36. Hajar T, Leshem YA, Hanifin JM, et al. A systematic review of topical corticosteroid withdrawal ("steroid addiction") in patients with atopic dermatitis and other dermatoses. J Am Acad Dermatol. 2015;72(3): 541-9.e2.

37. Litt JZ. Steroid-induced rosacea. Am Fam Physician. 1993;48(1):67-71.

38. Heibel HD, Hendricks AJ, Foshee JP, Shi VY. Rosacea associated with dupilumab therapy. J Dermatolog Treat. 2019;32(1):1-12. 